\title{
Hierarchical Linear Model of Monthly Rainfall with Regional and Seasonal Interaction Effects
}

\author{
Yonghua Zhu' ${ }^{1}$, Hongtao $\mathrm{Lu}^{1}$, Zilin Zhu' \\ ${ }^{1}$ Mathematics and physics department of North China Electric Power University, Beijing, China \\ ${ }^{2}$ Information Engineering Department of Huazhong University of Science and Technology, Wuhan, China \\ Email: zhuyh59@163.com,476502425@qq.com,363036808@qq.com
}

Received June, 2013

\begin{abstract}
According to the hierarchical characteristics of monthly rainfall in different regions, the paper takes the geographical factors and seasonal factors into the hierarchical linear model as the level effect. Through clustering methods we select two more representative regional meteorological data. We establish three-layer model by transforming the interactive structure date into nested structure data. According the model theory we perform the corresponding model calculations, optimization and analysis, accordingly to interpret the level effects, and residual test. The results show that most of the difference in Monthly Rainfall was respectively explained by Variables (Meteorological factors, seasonal effects, geographic effects) in different levels.
\end{abstract}

Keywords: Monthly Rainfall; Hierarchical Linear Model; Regional Effects; Interaction Effect Component

\section{Questions and Data Description}

For the defects of the past rainfall's regression, the literature [1] propose the regression model which take the factors and other effects into consideration. From the characteristics of monthly rainfall, we establish a twolayer model with the seasonal effect, in the model longitudinal data is grouped by month. Then through a series of operations, such as correlation analysis, data preprocessing, classification of seasonal effects, establishment of virtual indicators, gradually building models, the interpretation of fixed effects and random effects to complete the HLM2 model on monthly rainfall[1].

To dig the effect of monthly rainfall and various factors in different seasons and regions, we consider the data set: The meteorological data of Beijing, Tianjin and other 34 major cities in 1996-2009 (monthly rainfall/(mm), average temperature $/\left({ }^{\circ} \mathrm{C}\right)$, sunshine hours/(h), average relative humidity/(\%), average air pressure/(100pa), hereinafter referred to as rainfall, temperature, sunshine, humidity, pressure. To take Beijing and Nanjing for example, get the following figure.

Figure 1 shows that, the monthly precipitation curve showed two features: the two regions' data show a certain cycle as a unit of year; overall, the Beijing's rainfall is always greater than Nanjing's. Therefore, in the study of the differences of rainfall, we not only should consider the impact factors and seasonal effects, but also need to consider the regional differences. Based on the previous two-layer model, we attempt to establish a three-layer model on the effects of a regional group and seasons.

\section{Model and Analysis}

\subsection{Model}

Level 1 model: the regression between rainfall and temperature, sunshine, humidity, air pressure. Outcome variables $\mathrm{Yij}$ represents the rainfall of the month $\mathrm{j}$ of the year I $(i=1,2, \ldots 14, j=1,2, \ldots 12), x 1 \mathrm{ij}, x 2 \mathrm{ij}$, $x 3 \mathrm{ij}$, respectively, for the temperature, sunshine, humidity of the month $\mathrm{j}$ of the year $\mathrm{i}$.

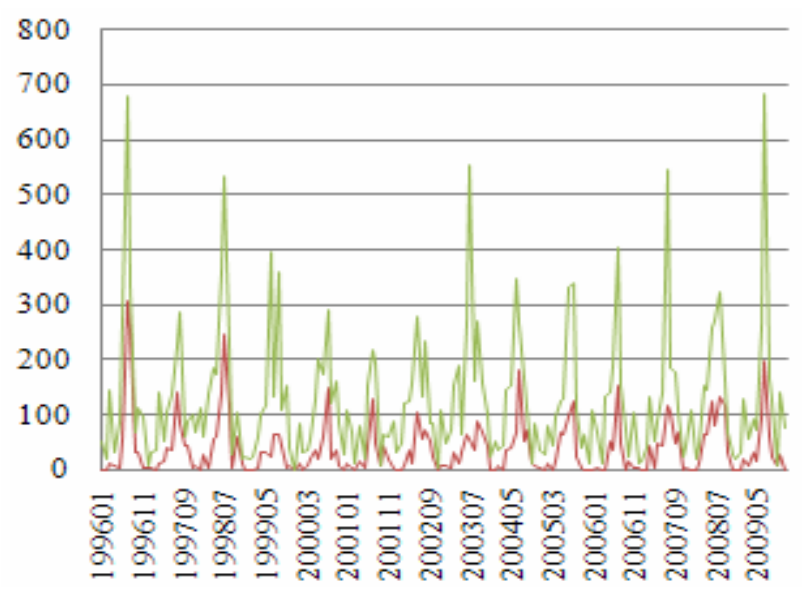

Figure 1. Beijing and Nanjing's monthly rainfall map(red: Beijing; blue: Nanjing). 
Level 2 model: Create two new virtual season index $\mathrm{CQ}, \mathrm{X}$, to distinguish three kinds of seasonal effects (Winter, Spring, Summer). The combination of their values and other factors indicate the slope of the temperature, sunshine and humidity with rainfall in different seasons.

Level 3 model: Establish geographical index to explain the intercept and slop Level 2 model (model about the relationship between season with the coefficient of Level 1). Comprehensive three-tier model, it express there is regression (intercept and slope) about different degrees of effect of various factors and seasonal precipitation in different regions.

Basic data includes 31 cities, its geographical spread and the seasonal variations are large. If we want to establish the index about geographical differences, which measure the monthly rainfall of 31 cities, and make three-tier regression. It would be difficult. If we want to establish virtual index, at least we should build five. But in the HLM2's level 2, the maximum number of considered geographical effects is five. Even have built a three-tier model, the fixed coefficients and random coefficient which need to test will be large (in Level 3, there will be 35 items including the intercept, slope, and random items), so effects analysis is not easy to make. If we take some quantitative methods (AHP, quantitative weighting, expert scoring method, etc) to establish a index which can unified measure geographical differences of 31 cities. It's more difficult and difficult to estimate accurately the extent, because qualitative indicators are always randomness and fuzziness. Making scientific analysis and rigorous validation to its quantitative is another major issue [3].
The two effects which affect the rainfall are seasonal effect and geographical effects. But we find there are three seasonal effects in geography, and in a season there are also two geographical effects, two effects are interaction effect, rather than simply "students- class- school" nested structure. In this case Raudenbush (1993) developed a method; Level 2 is the definition of "unit" effect which is classified by two interacted factors. Level 1 represents the link between variables under the influence of the "unit". This model has only two layers, known as Hierarchical Cross-classified Linear Model, HCM2 [2]. Taking rainfall for example, HCM model mainly research which independent variable the seasonal level and regional level have, and the characteristics of two- factor interactions between seasonal and regional levels.

Here we attempt to establish a three-layer model to decompose the interaction structure of two-factor. We do hierarchical processing to the interactive structure as follows.

In the level 1 the data set formed by this method reach 48 groups (12 months * 4 cities) by the effect of six seasons; In level 2 model 6 units are influenced by two geographical effects. The three-tier model is the same as the interaction effect model; each city's monthly data is corresponding to seasonal effects and regional effects.

List 1. The virtual index of seasonal effects in Level 2.

\begin{tabular}{lcc}
\hline \multicolumn{1}{c}{ Month } & CQ & X \\
\hline $12,1,2$ (winter) & 0 & 0 \\
$3,4,5,9,10,11$ (spring) & 1 & 0 \\
$6,7,8$ (summer) & 0 & 1
\end{tabular}

List 2. Data' two- factor interactions between seasonal and regional levels.

\begin{tabular}{ccccc}
\hline Region & Cities & Winter & Spring and Autumn & Summer \\
\hline \multirow{2}{*}{ North China } & Beijing & 1212 & 34591011 & 678 \\
& Tianjin & 1212 & 34591011 & 678 \\
\multirow{2}{*}{ East China } & Nanjing & 1212 & 34591011 & 678 \\
& Hefei & 1212 & 34591011 & 678 \\
\hline
\end{tabular}

List 3. Stratification of seasonal-geographical two-factor interacted structure.

\begin{tabular}{lll}
\hline Region & Season & City monthly weather data \\
\hline \multirow{2}{*}{ North China } & North-Winter & Beijing, Tianjin $(12,1,2)$ \\
& North Spring & Beijing, Tianjin $(3,4,5,9,10,11)$ \\
& North-Summer & Beijing, Tianjin $(6,7,8)$ \\
East China & East-Wpring & Nanjing, Hefei $(12,1,2)$ \\
& East-Summer & Nanjing, Hefei $(6,7,8)$ \\
\hline
\end{tabular}




\subsection{Zero Model}

Level 1 model:

$$
Y_{i j k}=P_{0 j k}+e_{i j k}
$$

Level 2 model:

$$
P_{0 j k}=B_{00 k}+r_{0 j k}
$$

Level 3 model:

$$
B_{00 k}=G_{000}+u_{00 k}
$$

$P_{0 j k}$ is the average monthly rainfall in the region $\mathrm{k}$ and season $\mathrm{j}, e_{i j k}$ is the individual differences of monthly rainfall at the same region and season. $B_{00 k}$ is the average of all average seasonal rainfalls at region $\mathrm{k}, r_{0 j \mathrm{k}}$ is the variational degree between different seasons at the same region. $G_{000}$ is the average rainfalls in all seasons at all regions, $u_{00 k}$ represent the variational relative to he mean at different regions. Zero model parameter estimation results are listed below.

Based on the principle of variance decomposition described above we can obtain follows, the group differences of monthly rainfall group differences account for $47.8 \%$, The differences of monthly rainfall affected by seasonal effect account for $42.2 \%$, regional impact account for $10.0 \%$ of the total differences. That shows the $52.2 \%$ differences of monthly rainfall are related to the geographical and seasonal effects. This suggest us we should add more explanatory variables to the level 1 and level 2 to explain more variance of levels.

\subsection{Random Effects Model}

Level 1 model:

$$
Y_{i j k}=P_{0 j k}+P_{1 j k} x_{1 i j k}+P_{2 j k} x_{2 i j k}+P_{3 j k} x_{3 i j k}+e_{i j k}
$$

Level 2 model:

$$
\begin{aligned}
& P_{0 j k}=B_{00 k}+r_{0 j k} \\
& P_{1 j k}=B_{10 k}+r_{1 j k} \\
& P_{2 j k}=B_{20 k}+r_{2 j k} \\
& P_{3 j k}=B_{30 k}
\end{aligned}
$$

Level 3 model:

$$
\begin{aligned}
& B_{00 k}=G_{000}+u_{00 k} \\
& B_{10 k}=G_{100} \\
& B_{20 k}=G_{200} \\
& B_{30 k}=G_{300}+u_{30 k}
\end{aligned}
$$

Compared to the zero model, the variance components of three-intercept of the random effects model were reduced by $18 \%, 11 \%, 17 \%$. Clearly, this variance is explained by the various factors added to the level 1 .

\subsection{Optimalizing Full Model}

Model Overview: the total number of level 1 units is $672=4$ cities $* 12$ months $* 14$ years (in addition to missing

\begin{tabular}{|c|c|c|c|c|}
\hline & Random effects & Standard deviation & Variance & $\mathrm{df}$ \\
\hline \multirow[t]{2}{*}{ Level 1} & Individual random effects & 40.64 & 1651.81 & -- \\
\hline & Level 1 intercept & $44.91 * *$ & 2017.75 & 46 \\
\hline \multirow[t]{2}{*}{ Level 2} & Temperature corresponds to the slop & $7.24 * *$ & 52.51 & 47 \\
\hline & Sunshine corresponds to the slop & $0.32 * *$ & 0.10 & 47 \\
\hline \multirow{2}{*}{ Level 3} & Level 2 intercept & $19.91 * *$ & 396.43 & 1 \\
\hline & The intercept of humidity corresponding to the slope & $1.19 * *$ & 1.42 & 1 \\
\hline
\end{tabular}
values, the total is 660); the total number of level 2 units is $48=4$ cities* 12 months, belonging to 48 different "regions- season"; the total number of level 3 units $2=2$ regions.

Level 1 model:

$$
Y_{i j k}=P_{0 j k}+P_{1 j k} x_{1 i j k}+P_{2 j k} x_{2 i j k}+P_{3 j k} x_{3 i j k}+e_{i j k}
$$

Level 2 model:

$$
\begin{aligned}
& P_{0 j k}=B_{00 k}+B_{01 k} C Q_{0 j k}+B_{02 k} X_{0 j k} \\
& P_{1 j k}=B_{12 k} X_{1 j k}+r_{1 j k} \\
& P_{2 j k}=B_{21 k} C Q_{2 j k}+r_{2 j k} \\
& P_{3 j k}=B_{31 k} C Q_{3 j k}+B_{32 j} X_{3 j k}
\end{aligned}
$$

List 4. Variance components' estimation of levels.

\begin{tabular}{cccc}
\hline Random effects & Standard error & Variance components & df \\
\hline Random item of Level1's intercept & $44.79 * *$ & 2006.39 & 46 \\
Random item of Level 1 & 47.65 & 2270.68 & -- \\
Random item of Level 3 & $21.84 * *$ & 477.12 & 1 \\
\hline
\end{tabular}

List 5 . The results of random effects models. 
Level 3 model:

$$
\begin{aligned}
& B_{00 k}=G_{001} D_{k} \\
& B_{01 k}=G_{010} \\
& B_{02 k}=G_{020}+u_{02 k} \\
& B_{12 k}=G_{120} \\
& B_{21 k}=G_{211} D_{k} \\
& B_{31 k}=G_{310}+G_{311} D_{k} \\
& B_{32 k}=G_{320}
\end{aligned}
$$

There are three parts affected by region in total: level 1 's intercept, sunshine slope in spring and autumn, Humidity slope in spring and autumn. In other words, the geographical differences are obvious in the overall mean. In the spring and autumn, the differences of the impact of humidity and sunshine to rainfall are significant [4].

In Figures 4-2 A stand for north, B stand for south, red stand for spring and autumn, blue stand for other seasons.

Obviously, there is a positive correlation between rainfall and humidity. In southern spring and autumn (chart B), humidity causes greater impact on rainfall.

In above figure, A stand for north, B stand for south, red stand for spring and autumn, blue stand for other seasons. There is a weak negative correlation between sunshine and rainfall in spring and autumn in two regions. The other seasons are messier and the relationship is unknown. The relationship can also be observed from the model and coefficients.

There is a great negative correlation between sunshine and rainfall in autumn. Other seasons were not significant and the north and south regions' differences were not significantly.

Compared to the zero models, level 1 model's random

\begin{tabular}{|c|c|c|c|c|}
\hline & Fixed effects & Coefficient & Standard error & df \\
\hline \multirow{3}{*}{ Level 1 intercept } & Intercept G001 & $45.55 * *$ & 3.19 & 652 \\
\hline & Winter and autumn G020 & $24.42 * *$ & 2.84 & 652 \\
\hline & Summer G030 & $112.08^{*}$ & 6.42 & 652 \\
\hline Temperature slope & Summer G130 & $-10.42 *$ & 4.38 & 47 \\
\hline \multirow[t]{2}{*}{ Sunshine slope } & Winter and autumn G221 & $-0.35^{*}$ & 0.15 & 47 \\
\hline & Winter and autumn G320 & $1.08 *$ & 0.51 & 652 \\
\hline \multirow[t]{2}{*}{ Humidity slope } & Winter and autumn G321 & $2.21^{*}$ & 1.01 & 652 \\
\hline & Summer G330 & $4.81 * *$ & 0.93 & 652 \\
\hline
\end{tabular}
item variance changes little. [5]The random items of level 2 and level 3 models are different, but we can obviously find that the vast majority of random effects are explained by different levels' seasonal and geographical variables, and random item's variance is very small.

List 6. The results of fixed effects models.
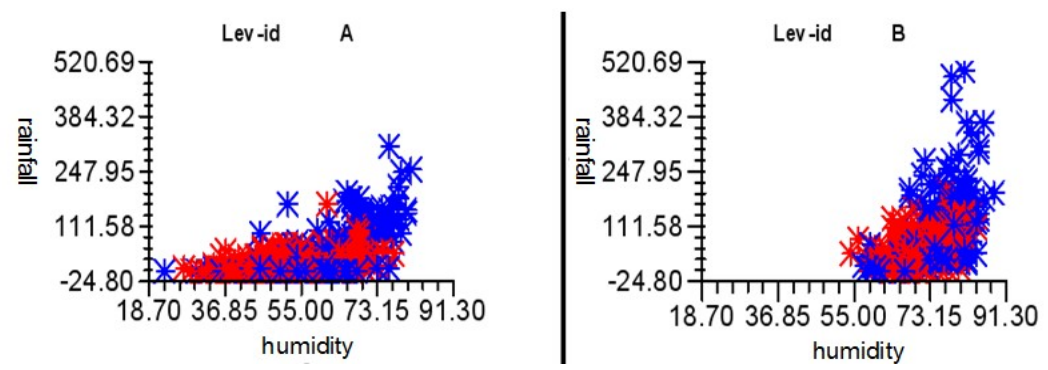

Figure 2. Scatter diagram about the relationship between humidity and rainfall in different regions.
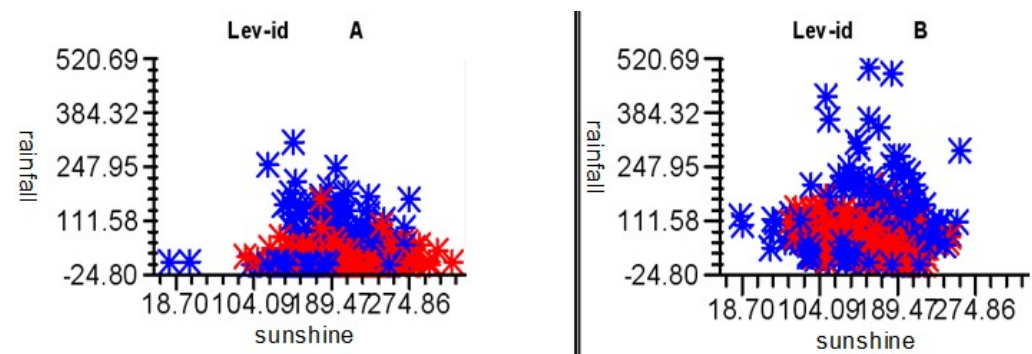

Figure 3. Scatter diagram about the relationship between sunshine and rainfall in different regions. 


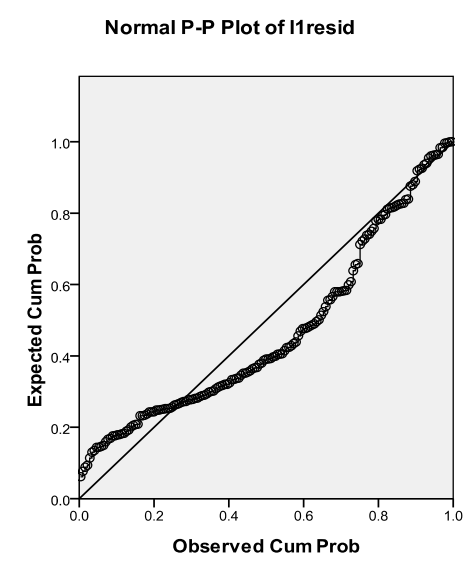

(a) Northern spring and autumn residuals

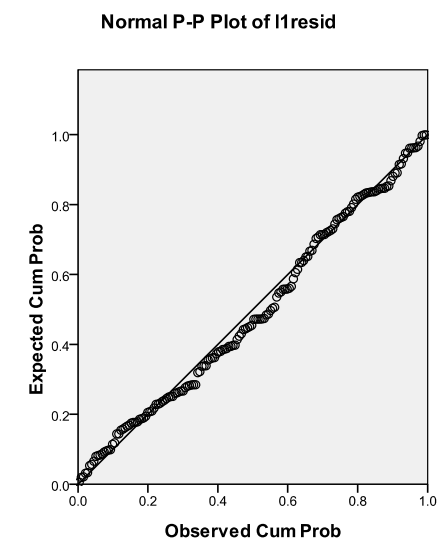

(c) Southern spring and autumn residuals
Normal P-P Plot of I1resid

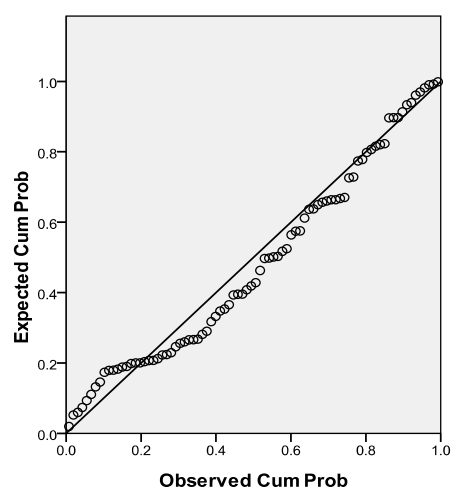

(b) Northern summer residuals

Normal P-P Plot of I1resid

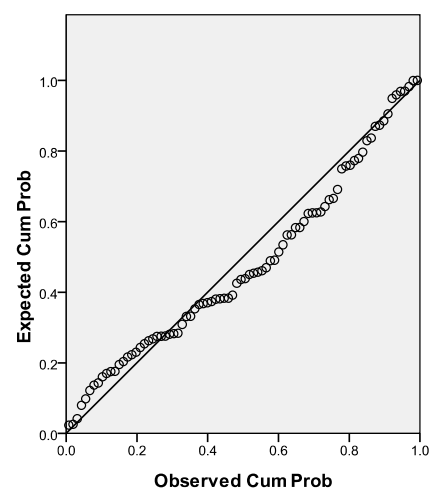

(d) Southern summer residuals

Figure 4. Level 1 model's residual comparison.

List 7. Variance components' estimation of levels.

\begin{tabular}{clccc}
\hline & \multicolumn{1}{c}{ Random effects } & Standard error & Variance & df \\
\hline Level 1 & Individual random effects & 42.63 & 1817.86 \\
\multirow{2}{*}{ Level 2 } & Temperature corresponds to the slop & Sunshine corresponds to the slop & $6.23^{* *}$ & 38.93 \\
Level 3 & Level 1's intercept corresponds to the slop in summer & $0.36^{* *}$ & 47 & 0.13 \\
4
\end{tabular}

List 8. HLM3 Model summary and comparison.

\begin{tabular}{lccc}
\hline & Model 1 (zero model) & Model 2 (random effects model) & Model 3 (final model) \\
\hline$\sigma 2$ & 2270.68 & 1651.81 & 1817.86 \\
Level-2: & - & -- & -- \\
$\mu 00 \mathrm{p}$ & 2006.40 & 2017.75 & -- \\
$\mu 11 \mathrm{p}$ & -- & 52.52 & 38.93 \\
$\mu 22 \mathrm{p}$ & -- & 0.10 & 0.13 \\
Level-3: & -- & -- & -- \\
$\mu 00 \mathrm{~b}$ & 477.12 & 396.44 & - \\
$\mu 02 \mathrm{~b}$ & -- & -- & 55.86 \\
$\mu 03 \mathrm{~b}$ & -- & 1.43 & -- \\
Total deviation & 7100.66 & 6936.67 & 6865.15 \\
Number of Parameters & 4 & 10 & 12 \\
Iterations & 4 & 512 & 673 \\
The total variance & 4754.20 & -- & -- \\
\hline
\end{tabular}




\section{Model Summary}

\subsection{Model Comparison}

Compared with the zero models, the random item's variance of level 1 changes little. The random items in level 2 and level 3 can't be comparable. But we can obviously find that the vast majority of random effects are explained by different levels' seasonal and geographical variables, and random item's variance is very small. Hierarchical interpretation of the effect of rainfall is significant, and seasonal and geographical explanatory variables play a good role in regression.

\subsection{Residual Analysis}

From the above residual plots we can get two features: Overall, the residuals of the south are closer to the normal distribution than those of the north, and spring season is closer to the normal distribution than summer season, because the southern seasonal effect and geographical effect are more significant than the northern on the whole, and the summer's effect is more significant than the winter and autumn's. This result the differences of rainfall in the South in summer has been more fully explained, assumption of the residuals closer to the normality.

Overall, HLM3 model's size of the model residuals is similar to the HLM2 models, the summer's residuals are bigger, but their relative offset is to a lesser extent.

\section{HLM3 Model Conclusions}

In this paper, we mainly research the rainfall HLM3 model under the seasonal and geographical effects. Here is a brief summary of geographical effects.

From the overall average level, the geographical differences of monthly rainfall are significant.

Most of monthly rainfall's differences between the groups can be explained by seasonal and geographical of variable levels.

In spring and autumn the degree of humidity and sunshine's influence on the rainfall has significant differences. Positive correlation with precipitation and humidity, sunshine is a negative correlation;

In the summer there is a big negative correlation between temperature and precipitation, but in the other seasons it's not significant and the geographical differences are obvious.
From the data fit through the hierarchical model, although larger residuals in summer, its relative deviation is more minimum than the other seasons; the overall fit of the South is better than the North.

\section{Summary}

After taking different regions of precipitation into comparison, we take the effect of geographical factors as a higher level. Using cluster analysis, we select two regional more representative meteorological data. Translate structure of the interaction data into nested structure, and establish a corresponding three-level linear model (HLM3). In accordance with model theory we do the corresponding model calculation, optimization and analysis and reach some major conclusions. The explanatory variables of various levels (the meteorological factors, seasonal effects, geographic effects) can well explained the differences in monthly rainfall.

Hierarchical linear models have been widely used in social science fields. About the natural science problems we can make use of the professional knowledge and draw on some ideas and methods of appropriate social science to establish an appropriate model. With the development of a variety of technologies, in most cases the size of data will no longer be limited, a lot of data can be repeatedly observed and recorded, which cause the formation of the corresponding longitudinal data. Therefore, the hierarchical linear model will be widely used.

\section{REFERENCES}

[1] Y. H. Zhu and G. X. Jiang, "Hierarchical Linear Model and Its Research on Hierarchical Characteristics of Rainfall," 2011 International Conference on Multimedia Technology (ICMT 2011), pp. 2146-2150.

[2] S. W. Raudenbush, A. S. Bryk and Z. G. Guo, "Hierarchical Linear Models: Application and Data Analysis Method," Beijing: Social Sciences Academic Press, 2007, pp. 83-90.

[3] J. C. Wang, H. Y. Xie and B. F. Jiang, "Application of Hierarchical Linear Model-Methods and Applications," Beijing: Higher Education Press, 2007, pp. 27-30.

[4] L. Zhang, L. Lei and B. L. Guo, "Application of Hierarchical Linear Model," Beijing: Science and Education Press, 2003, pp. 28-40.

[5] X. Zhang and J. Y. Wang, "The Study for the Sample Size Problem about Hierarchical Linear Models," Statistics and Decision, Vol. 15, 2010, pp. 4-8. 